\title{
Impact of coronavirus disease 2019 (COVID- 19) pandemic on attitude, behavior, and mental health of patients with rheumatic diseases
}

\author{
Marwa A. H. Hammad' (D), Mervat Eissa ${ }^{2 *}$ and Ghada A. Dawa ${ }^{1}$ (D)
}

\begin{abstract}
Background: The coronavirus disease 2019 (COVID-19) pandemic has become a global health, social, and economic crisis. Healthcare professionals, patients, healthy individuals, and the whole community are under inevitable psychological pressure which may cause different psychological problems as fear, anxiety, depression, and insomnia. The aim was to assess the impact of the COVID19 pandemic on the attitude, behavior, and mental health of rheumatic patients and to compare them with healthy individuals. This is a case-control study, 360 participants were included and divided into a patient group composed of 180 patients with rheumatic diseases, and a control group composed of 180 healthy people. Data were collected via a self-administered structured questionnaire designed on Google forms. It was sent to participants via social networks and emails to different rheumatic patients and healthy individuals. Mental health was measured by the 5-item Brief Symptom Rating Scale (BSRS-5).

Results: The mean age of cases and control were (35.05 \pm 8.79 vs $34.56 \pm 9.06)$ years. In comparing attitudes and behavior toward COVID 19, there was a statistically significant difference $(p \leq 0.05)$ between both groups regarding washing hands, going outdoors, wearing masks and gloves outdoors, and staying in their rooms. Patients depended mainly on telehealth more than usual where about $50 \%$ used either phone calls, internet or sent their relatives to their physicians; moreover, $20 \%$ did not contact their physicians at all the past few months. There was a statistically significant difference ( $p \leq 0.05$ ) between both groups regarding feeling angry/irritated, inferior and insomniac. The BSRS-5 total score and being defined as a psychiatric case (according to the BSRS-5 scale) also differed significantly between patients and controls. Systemic lupus erythematosus (SLE) patients showed more adherence to their medications and stayed mostly at home and they have higher BSRS scores.

(Continued on next page)
\end{abstract}

\footnotetext{
* Correspondence: mervateissa@kasralainy.edu.eg

${ }^{2}$ Rheumatology Department, Faculty of Medicine, Cairo University, Cairo,

Egypt

Full list of author information is available at the end of the article
}

\section{Springer Open}

(c) The Author(s). 2020 Open Access This article is licensed under a Creative Commons Attribution 4.0 International License, which permits use, sharing, adaptation, distribution and reproduction in any medium or format, as long as you give appropriate credit to the original author(s) and the source, provide a link to the Creative Commons licence, and indicate if changes were made. The images or other third party material in this article are included in the article's Creative Commons licence, unless indicated otherwise in a credit line to the material. If material is not included in the article's Creative Commons licence and your intended use is not permitted by statutory regulation or exceeds the permitted use, you will need to obtain permission directly from the copyright holder. To view a copy of this licence, visit http://creativecommons.org/licenses/by/4.0/. 


\begin{abstract}
(Continued from previous page)
Conclusion: Patients with rheumatic diseases show comparable degrees of anxiety and depression to healthy individuals, but higher distress symptoms and panic in the form of anger, irritability, and insomnia. They have a significantly higher sense of inferiority and a higher total BSRS compared to controls. SLE patients show more adherence to their medications and stay mostly at home as a reflection of feeling more vulnerable. Moreover, they have higher degrees of psychological affection in the form of higher BSRS scores. Abandoning drug purchasing without medical prescription is necessary in Egypt to protect our patients from unnecessary drug shortages adding to their fear and anxiety. Mental health should be addressed in the same manner we deal with the infectious disease itself, being of no less importance. Mental health professionals, social workers, and support groups need to provide psychological support to vulnerable populations, including patients with rheumatic diseases.

Rheumatologists should be aware of the need for psychiatric consultation for their patients whenever necessary.
\end{abstract}

Keywords: COVID-19, Rheumatic patients, Attitude, Behavior, Mental health

\section{Background}

The corona virus disease 2019 (COVID-19) is an infectious disease caused by the severe acute respiratory syndrome coronavirus 2 (SARS-CoV-2), which originated in Wuhan, China, in early December 2019 and declared a pandemic by the World Health Organization (WHO) on 11 March 2020 [1, 2]. Previous data from similar outbreaks, such as the Severe Acute Respiratory Syndrome [SARS], showed that the community suffered considerable anxiety, depression, and panic, resulting in a significant psychological impact [3-5].

The mortality of COVID-19 is even higher than previous epidemics inducing profound fear [6,7]; mental health reflections can last longer and even have a higher frequency than the epidemic itself $[8,9]$. Shortages of masks and health equipment, quarantine, lack of effective treatment, closure of schools and public places, boredom, fear of infection, or death could increase panic. Moreover, it can lead to a feeling of insecurity due to economic and social losses [10-14].

Patients with rheumatic diseases-those suffering from rheumatoid arthritis, systemic lupus, scleroderma, ankylosing spondylitis, Sjogren's disease, inflammatory myositis, fibromyalgia, or others-are considered at greater risk both due to their chronic illnesses and use of immunosuppressive drugs [15]. They are also facing many issues- drug shortages, difficulties reaching clinics or hospitals, and hazards of physical inactivity staying at home. It is expected that these individuals with chronic diseases will be prone to higher levels of impaired psychology [16] since the COVID-19 tends to hit hardest those with multiple comorbidities [17]. The American College of Rheumatology [ACR] Issues COVID-19 Treatment Guidance for Rheumatic Disease Patients recommended the reduction of the number of health care encounters; laboratory monitoring less frequently, using telehealth, and increasing intervals between intravenous doses and they set the basis for when to start, stop, or reduce medications [18].
A recent public survey conducted in China about the psychological impact of the COVID-19 outbreak showed that about a third of participants experienced moderate to severe anxiety and about half experienced moderate to severe psychological impairment [19]. No previous researches studied the psychological impact of COVID-19 on patients with rheumatic diseases in Egypt. This present study represents the first mental health survey conducted in Egypt addressing this vulnerable population. From this point we aimed to assess the impact of [COVID-19] pandemic on the attitude, behavior, and mental health of patients with rheumatic disease and to compare them with healthy individuals.

\section{Methods \\ The study design}

This is an Egyptian case-control study, sample size was calculated based on findings from the pilot study prevalence among cases $17 \%$ and prevalence among control $40 \%$ case to control 1:1. A total of 360 participants were included consecutively in this study and divided into two groups: a patient group composed of 180 patients with rheumatic diseases and a control group composed of 180 healthy individuals. Rheumatic patients and healthy individuals had been recruited from 14 to 29 April 2020. Data were collected via a self-administered structured questionnaire that were delivered as a Google form in Arabic, which were sent to participants via social networks targeting rheumatic patients and healthy individuals, or via emails. The survey was translated into Arabic [mother tongue of Egyptians] and validated by the authors. It took about 5-10 min to be completed. The participants' information is kept anonymous. The translated form is available in the supplemental files. All control subjects were age, sex, socio-economically, and geographically matched with the patients [living allover Egypt those educated enough to use social media and internet]. The study complied with the Declaration of Helsinki, and informed online consent has been obtained 
from the subjects. Ethical approval was taken from Institutional Review Board IRB under number of (ZU-IRB \#6096).

\section{The questionnaire}

It was designed as a self-administered questionnaire with closed questions [yes/no questions, rating scale, and multiple-choice questions] and some open-ended questions. It was composed of 7 parts and a total of 37 questions.

1. Description of the survey, its aim, targeted population, and time needed to fill it.

2. Participants' characteristics, including age, gender, nationality, residential area, smoking, infection with COVID19, defining his/her rheumatic disease, and previous lung affection.

3. A question about the most reliable information source about COVID19 they relied on TV/radio, internet, social media groups, own physicians, friends, and family.

4. Five questions about their attitude towards some protective measures [such as staying at home, wearing protective masks, wearing gloves, washing hands with water and soap, eating a healthy diet and exercises help]; answers were "yes," "no," or "maybe"

5. Five questions about their behavior [going outdoors, wearing masks outdoors and wearing gloves outdoors, spending much time in the room away from the family] and frequency of washing hands]; graded as a Likert-type scale "never," "a bit of the time/few times," "most of the time", and "always" based on the question.

6. Eight questions for rheumatic patients only about: contact with the physician, the means and causes of contact and their feeling afterward, their attitude towards immunosuppressive drugs.

7. Six questions for mental health assessment for patients and controls: Mental health was measured by the 5-item Brief Symptom Rating Scale [BSRS-5] which was derived from the 50-item BSRS [20]. This effective screening instrument has satisfactory reliability and validity and is widely used to identify psychiatric impairment in medical settings and community samples [20-22]. The scale was translated and back-translated to Arabic language and a pilot study was conducted to test the applicability and the clarity of the scale then changes were made accordingly. It consisted of five symptom items, namely:

- Feeling tense or high-strung [anxiety];

- Feeling depressed or in a low mood [depression];

- Feeling easily annoyed or irritated [hostility];
- Feeling inferior to others [inferiority];

- Having trouble falling asleep [insomnia];

The score for each item ranges from 0 to $4[0$, not at all; 1 , a little bit; 2 , moderately; 3 , quite a bit; and 4, extremely], based on a 5-point Likert-type scale, participants were asked to indicate how much discomfort they experienced that was caused by a particular symptom in the past week [including the current day]. The sum of the scores across the five items represented the total score of the BSRS-5 and ranged from 0 to 20 . A total score on the BSRS-5 above 14 may indicate a severe mood disorder. Scores between 10 and 14 may indicate moderate mood disorders, and those between 6 and 9 could indicate mild mood disorders. A cut-off score of $\geq$ 6 was considered as a BRSR-5 defined psychiatric case [20].

\section{Statistical analysis}

The collected data were statistically analyzed using the Statistical Package for Social Science software [SPSS] [Version 20.0. Armonk, NY: IBM Corp.]. Continuous variables with a normal distribution were reported as mean and standard deviation [SD]. Categorical variables were summarized as frequencies and percentages. Quantitative data were evaluated using Independent $t$ test or one-way ANOVA, which were suitable for normally distributed data, while qualitative data were evaluated by chi-square test $\left[\chi^{2}\right]$ or Fisher's exact test. $p$ values $\leq$ $0.05^{*}$ and $\leq 0.001^{* *}$ were considered statistically significant and highly statistically significant, respectively.

\section{Results}

Socio-demographic and personal characteristics of the studied groups showed that there was no statistically significant difference [ $p>0.05]$ between both groups; ensuring their comparability. The mean age of cases and control groups were $35.05 \pm 8.79$ vs $34.56 \pm 9.06$ years with the highest proportion of them were females $[78.3 \%$ vs $77.8 \%$ ], non-smokers [ $83.3 \%$ vs $86.7 \%$ ] and all of them were non-infected with COVID and about $48.9 \%$ vs $47.2 \%$ received their COVID information from social media. About $40 \%$ of the case group had RA and the majority of this group [78.3\%] did not have previous lung affection (Table 1).

On comparing attitude and behavior toward COVID19 pandemic among the studied groups, no statistically significant difference $[p>0.05]$ was found between patients and the control group regarding their belief that staying at home protects them from infection, healthy diet and exercises help, and frequent washing hands, while there was a statistically significant difference $[p \leq$ 0.05 ] between both groups regarding their behavior regarding washing hands, going outdoors, wearing masks 
Table 1 Socio demographic and personal characteristics of the studied groups ( $n=360)$

\begin{tabular}{|c|c|c|c|c|}
\hline Characteristics & $\begin{array}{l}\text { Cases } \\
(n=180)\end{array}$ & $\begin{array}{l}\text { Controls } \\
(n=180)\end{array}$ & Test & $p$ value \\
\hline \multicolumn{5}{|l|}{${ }_{c \text { d Age (years) }}$} \\
\hline Mean \pm SD & $35.05 \pm 8.79$ & $34.56 \pm 9.06$ & ${ }^{\mathrm{a}} 0.526$ & 0.600 \\
\hline \multicolumn{5}{|l|}{${ }^{c}{ }^{d}$ Sex no (\%) } \\
\hline Female & $141(78.3 \%)$ & $140(77.8 \%)$ & $\mathbf{b}_{0.016}$ & 0.899 \\
\hline Male & 39 (21.7\%) & $40(22.2 \%)$ & & \\
\hline \multicolumn{5}{|l|}{ Smoking, no (\%) } \\
\hline No & $150(83.3 \%)$ & $156(86.7 \%)$ & $\mathbf{b}_{0.784}$ & 0.376 \\
\hline Yes & $30(16.7 \%)$ & $24(13.3 \%)$ & & \\
\hline \multicolumn{5}{|c|}{ Infected with COVID, no (\%) } \\
\hline No & $180(100 \%)$ & $180(100 \%)$ & - & - \\
\hline Yes & $0.0(0 \%)$ & $0.0(0 \%)$ & & \\
\hline \multicolumn{5}{|c|}{ COVID information source, no (\%) } \\
\hline TV/radio & $39(21.7 \%)$ & $41(22.8 \%)$ & $\mathbf{b}_{5.926}$ & 0.205 \\
\hline Internet & $42(23.3 \%)$ & $44(24.4 \%)$ & & \\
\hline Social media & $88(48.9 \%)$ & $85(47.2 \%)$ & & \\
\hline My physician & $9(5 \%)$ & $3(1.7 \%)$ & & \\
\hline Friends and family & $2(1.1 \%)$ & $7(3.9 \%)$ & & \\
\hline
\end{tabular}

Rheumatological disease, no (\%)

\begin{tabular}{ll} 
RA & $72(40 \%)$ \\
SLE & $67(37.2 \%)$ \\
Rhupus & $4(2.2 \%)$ \\
AS & $9(5 \%)$ \\
Behcet & $12(6.7 \%)$ \\
Sjogren & $2(1.1 \%)$ \\
Vasculitis & $1(0.6 \%)$ \\
Fibromyalgia & $5(2.8 \%)$ \\
Polymyalgia & $1(0.6 \%)$ \\
APA & $1(0.6 \%)$ \\
Sarcoidosis & $1(0.6 \%)$ \\
IBD & $1(0.6 \%)$ \\
Scleroderma & $1(0.6 \%)$ \\
DM & $1(0.6 \%)$ \\
PSA & $1(0.6 \%)$ \\
Mixed & $1(0.6 \%)$ \\
Lung affection, no (\%) & \\
No & $141(78.3 \%)$ \\
Yes & $39(21.7 \%)$ \\
\hline
\end{tabular}

COVID-19 coronavirus disease 2019, TV television, $R A$ rheumatoid arthritis, SLE systemic lupus erythematosus, $A S$ ankylosing spondylitis, FMS fibromyalgia, $A P A$ antiphospholipid syndrome, IBD inflammatory bowel syndrome, DM dermatomyositis, PSA psoriatic arthritis

${ }^{\mathrm{a}}$ Independent $t$ test

${ }^{\mathrm{b}} \mathrm{Chi}$ square test $\left(x^{2}\right)$

c Significant between RA, SLE, others

${ }^{\mathrm{d}}$ Significant between RA, SLE and gloves outdoors, and staying in their room (Table 2).

Mental health comparison among the studied groups showed that no statistically significant difference $[p>$ 0.05 ] was found between the patients and control groups regarding anxiety and depression, while there was a statistically significant difference $[p \leq 0.05]$ between both groups regarding feeling angry/irritated, inferior, and insomniac. BSRS- 5 total score and being defined as a psychiatric case according to BSRS-5 scale was significantly higher in patients (Table 3).

Regarding patients, attitude, and behavior towards their illness, medications, and medical advice, the highest proportion of them answered that they felt at higher risk and they felt no change in their disease activity; however, they believed that fear may increase disease activity. They contacted their physicians less than usual, mainly using the internet. They mainly asked for medication advice and most of them still felt non-assured after communication. Most of them had no fears about drugs, did not reduce doses/stop drugs, and many experienced drug shortages (Table 4).

On assessing the relationship between different parameters and patients' mental health degree, there was no statistically significant association $[p>0.05]$ between the degree of impaired mental health and all studied parameters except for "feeling at risk more than other" where there was a statistically significant association $[p \leq 0.05]$ where their feeling of "being at risk" was associated with a higher degree of impaired mental health [BSRS-5 $\geq 6$ ] (Tables 5 and 6).

Comparison between patients with rheumatoid arthritis (RA), systemic lupus erythematosus (SLE), and other rheumatic diseases regarding different parameters showed that there was a statistically significant difference $[p \leq 0.05]$ between them as regards age, sex, their belief that a healthy diet and exercises may help, frequency of going outdoors, BSRS-5 total score, being at risk, their feeling after communication, stopping drugs and experiencing drug shortages. RA patients had higher mean age and a higher percent of the male sex, they believe that a healthy diet and exercises can help, they go out only for necessities, they feel "maybe/not at risk", they feel less/the same anxiety and fear after communicating with their physicians, they are less adherent to some drugs and experience more drug shortages. SLE patients had a higher percentage of the female sex, they believe a healthy diet and exercises "maybe/not help," mostly "never go outdoors," they feel "yes at risk," feel more/the same anxiety, and fear after communication, and most do not stop drugs. Patients with other rheumatic diseases had higher mean BSRS-5 a total score and mostly "go outdoors freely any time," feel "not at risk," and experienced no drug shortage. 
Table 2 Attitude and behaviour regarding COVID among the studied groups $(n=360)$

\begin{tabular}{|c|c|c|c|c|}
\hline \multirow{2}{*}{$\begin{array}{l}\text { Protective attitude/ } \\
\text { behavior }\end{array}$} & \multirow{2}{*}{$\begin{array}{l}\text { Cases }(n=180) \\
\text { No }(\%)\end{array}$} & \multirow{2}{*}{$\begin{array}{l}\text { Controls }(n=180) \\
\text { No }(\%)\end{array}$} & \multirow[t]{2}{*}{${ }^{\text {a } \text { Test }}$} & \multirow[t]{2}{*}{$p$ value } \\
\hline & & & & \\
\hline \multicolumn{5}{|l|}{ Staying at home helps } \\
\hline No & $18(10 \%)$ & $9(5 \%)$ & 3.246 & 0.197 \\
\hline Yes & $98(54.4 \%)$ & $103(57.2 \%)$ & & \\
\hline Maybe & $64(35.6 \%)$ & $68(37.8 \%)$ & & \\
\hline \multicolumn{5}{|l|}{ Wearing masks helps } \\
\hline No & $b_{52}(28.9 \%)$ & $20(11.1 \%)$ & 19.85 & $<0.001^{* *}$ \\
\hline Yes & $57(31.7 \%)$ & $85(47.2 \%)$ & & \\
\hline Maybe & $71(39.4 \%)$ & 75 (41.7\%) & & \\
\hline \multicolumn{5}{|l|}{ Wearing gloves helps } \\
\hline No & b79 (43.9\%) & $44(24.4 \%)$ & 18.16 & $<0.001 * *$ \\
\hline Yes & $36(20 \%)$ & $64(35.6 \%)$ & & \\
\hline Maybe & $65(36.1 \%)$ & $72(40 \%)$ & & \\
\hline \multicolumn{5}{|l|}{ Washing hands helps } \\
\hline No & $3(1.7 \%)$ & $1(0.6 \%)$ & 10.46 & $0.005^{*}$ \\
\hline Yes & ${ }^{b} 129(71.7 \%)$ & $154(85.6 \%)$ & & \\
\hline Maybe & $48(26.7 \%)$ & $25(13.9 \%)$ & & \\
\hline \multicolumn{5}{|c|}{${ }^{c}{ }^{d}$ Healthy diet and exercises help } \\
\hline No & $21(11.7 \%)$ & $16(8.9 \%)$ & 1.071 & 0.585 \\
\hline${ }^{c}{ }^{\text {Yyes }}$ & $95(52.8 \%)$ & $103(57.2 \%)$ & & \\
\hline${ }^{\mathrm{c}}$ May be & $64(35.6 \%)$ & $61(33.9 \%)$ & & \\
\hline \multicolumn{5}{|l|}{ 'Going outdoors } \\
\hline${ }^{\mathrm{c}}$ Never & $48(26.7 \%)$ & $24(13.3 \%)$ & 12.64 & $0.002^{*}$ \\
\hline 'Only for necessities & $\mathrm{b}_{113}(62.8 \%)$ & $143(79.4 \%)$ & & \\
\hline Go out freely & $19(10.6 \%)$ & $13(7.2 \%)$ & & \\
\hline \multicolumn{5}{|l|}{ Wearing mask outdoors } \\
\hline Never & $66(36.7 \%)$ & $54(30 \%)$ & 11.82 & $0.019^{*}$ \\
\hline Few times & ${ }^{b} 28(15.6 \%)$ & $44(24.4 \%)$ & & \\
\hline Most of the time & $31(17.2 \%)$ & $43(23.9 \%)$ & & \\
\hline Always & $22(12.2 \%)$ & $22(12.2 \%)$ & & \\
\hline I do not go out & b33 (18.3\%) & $17(9.4 \%)$ & & \\
\hline \multicolumn{5}{|l|}{ Wearing gloves outdoors } \\
\hline Never & ${ }^{b} 90(50 \%)$ & $70(38.9 \%)$ & 19.33 & $0.001^{* *}$ \\
\hline Few times & ${ }^{b} 21(11.7 \%)$ & $41(22.8 \%)$ & & \\
\hline Most of the time & $20(11.1 \%)$ & $27(15 \%)$ & & \\
\hline Always & $14(7.8 \%)$ & $25(13.9 \%)$ & & \\
\hline I do not go out & $35(19.4 \%)$ & $17(9.4 \%)$ & & \\
\hline \multicolumn{5}{|l|}{${ }^{\mathrm{d}}$ Staying in your room } \\
\hline${ }^{\mathrm{d}}$ Move freely at home & $133(73.9 \%)$ & $134(74.4 \%)$ & 10.11 & $0.018^{*}$ \\
\hline A bit of time & ${ }^{b} 19(10.6 \%)$ & $33(18.3 \%)$ & & \\
\hline Most of time & $26(14.4 \%)$ & $13(7.2 \%)$ & & \\
\hline All of time & $2(1.1 \%)$ & $0.0(00 \%)$ & & \\
\hline \multicolumn{5}{|c|}{ Frequency of washing hands } \\
\hline Less than 5 times & $31(17.2 \%)$ & $26(14.4 \%)$ & 1.942 & 0.379 \\
\hline 5 to 10 times & $97(53.9 \%)$ & $90(50 \%)$ & & \\
\hline More than 10 times & $52(28.9 \%)$ & $64(35.6 \%)$ & & \\
\hline
\end{tabular}

${ }^{a}$ Chi square test $\left(x^{2}\right)$

${ }^{\mathrm{b}}$ Cell of significance

'Significant between RA, SLE, others

dSignificant between RA, SLE. ${ }^{*} P$ value is significant. ${ }^{* *} P$ value is highly significant 
Table 3 Mental health comparison among the studied groups $(n=360)$

\begin{tabular}{|c|c|c|c|c|}
\hline Mental health & $\begin{array}{l}\text { Cases }(n=180) \text {, } \\
\text { no }(\%)\end{array}$ & $\begin{array}{l}\text { Controls }(n=180) \text {, } \\
\text { no }(\%)\end{array}$ & Test & $p$ value \\
\hline \multicolumn{5}{|l|}{ Anxiety } \\
\hline Not at all & 17 (9.4\%) & $11(6.1 \%)$ & ${ }^{\mathrm{a}} 9.028$ & 0.060 \\
\hline A little bit & 34 (18.9\%) & $36(20 \%)$ & & \\
\hline Moderately & $54(30 \%)$ & 64 (35.6\%) & & \\
\hline Quite a bit & 49 (27.2\%) & $58(32.2 \%)$ & & \\
\hline Extremely & $26(14.4 \%)$ & $11(6.1 \%)$ & & \\
\hline \multicolumn{5}{|l|}{ Depression } \\
\hline Not at all & $28(15.6 \%)$ & 37 (20.6\%) & ${ }^{\mathrm{a}} 6.405$ & 0.171 \\
\hline A little bit & $44(24.4 \%)$ & 45 (25\%) & & \\
\hline Moderately & $41(22.8 \%)$ & $50(27.8 \%)$ & & \\
\hline Quite a bit & 44 (24.4\%) & $36(20 \%)$ & & \\
\hline Extremely & $23(12.8 \%)$ & $12(6.7 \%)$ & & \\
\hline \multicolumn{5}{|l|}{ Angry/irritated } \\
\hline Not at all & $4(2.2 \%)$ & $10(5.6 \%)$ & ${ }^{\mathrm{a}} 10.73$ & $0.030 *$ \\
\hline A little bit & $32(17.8 \%)$ & 44 (24.4\%) & & \\
\hline Moderately & 47 (26.1\%) & $58(32.2 \%)$ & & \\
\hline Quite a bit & ${ }^{\circ} 59(32.8 \%)$ & 42 (23.3\%) & & \\
\hline Extremely & 38 (21.1\%) & $26(14.4 \%)$ & & \\
\hline \multicolumn{5}{|l|}{ e'Inferiority } \\
\hline${ }^{\mathrm{e}}$ Not at all & ${ }^{`} 63(35 \%)$ & $108(60 \%)$ & ${ }^{\mathrm{a}} 41.20$ & $<0.001 * *$ \\
\hline A little bit & 39 (21.7\%) & 43 (23.9\%) & & \\
\hline Moderately & 35 (19.4\%) & 22 (12.2\%) & & \\
\hline Quite a bit & 25 (13.9\%) & $3(1.7 \%)$ & & \\
\hline Extremely & $18(10 \%)$ & $4(2.2 \%)$ & & \\
\hline \multicolumn{5}{|l|}{ Insomnia } \\
\hline Not at all & 20 (11.1\%) & 36 (20\%) & & \\
\hline A little bit & ${ }^{c} 25$ (13.9\%) & 60 (33.3\%) & & \\
\hline Moderately & 43 (23.9\%) & 43 (23.9\%) & & \\
\hline Quite a bit & 52 (28.9\%) & 27 (15\%) & & \\
\hline Extremely & 40 (22.2\%) & $14(7.8 \%)$ & a 39.41 & $<0.001 * *$ \\
\hline \multicolumn{5}{|l|}{ d e BSRS-5 total score } \\
\hline Mean \pm SD & $10.45 \pm 4.22$ & $8.16 \pm 3.74$ & $b_{5.466}$ & $<0.001 * *$ \\
\hline$<6$ not psychiatric case & $26(14.4 \%)$ & $45(25 \%)$ & ${ }^{\mathrm{a}} 6.334$ & $0.012^{*}$ \\
\hline$\geq 6$ psychiatric case & $154(85.6 \%)$ & $135(75 \%)$ & & \\
\hline \multicolumn{5}{|l|}{ Your fear about } \\
\hline Infection & $26(14.4 \%)$ & $15(8.3 \%)$ & ${ }^{a} 57.30$ & $<0.001 * *$ \\
\hline Family & ${ }^{c} 92(51.1 \%)$ & $136(75.6 \%)$ & & \\
\hline Quarantine & $6(3.3 \%)$ & $15(8.3 \%)$ & & \\
\hline Financial problems & $14(7.8 \%)$ & $14(7.8 \%)$ & & \\
\hline Medications & $11(6.1 \%)$ & $0.0(00 \%)$ & & \\
\hline Mixed fears & 31 (17.2\%) & 0.0 (00\%) & & \\
\hline
\end{tabular}

${ }^{a}$ Chi square test $\left(x^{2}\right)$

${ }^{\mathrm{b}}$ Independent $t$ test

${ }^{c}$ Cell of significance

${ }^{\mathrm{d}}$ Significant between RA, SLE, others

${ }^{\text {e}}$ Significant between RA, SLE. ${ }^{*} P$ value is significant. ${ }^{* *} P$ value is highly significant 
Table 4 Patients' attitude and behavior regarding disease, medications and medical advice $(n=180)$

\begin{tabular}{|c|c|c|}
\hline \multirow[t]{2}{*}{ Attitude/behavior } & \multicolumn{2}{|c|}{ Value $(n=180)$} \\
\hline & No & (\%) \\
\hline \multicolumn{3}{|l|}{${ }^{\mathrm{a} b}$ Are you at risk } \\
\hline No & 23 & 12.8 \\
\hline a byes & 97 & 53.9 \\
\hline a baybe & 60 & 33.3 \\
\hline \multicolumn{3}{|l|}{ Current disease activity } \\
\hline Decreased & 6 & 3.3 \\
\hline Did not change & 131 & 72.8 \\
\hline Increased & 43 & 23.9 \\
\hline \multicolumn{3}{|l|}{ Disease activity may increase due to } \\
\hline Fear & 92 & 51.1 \\
\hline Postponed follow-up visits & 22 & 12.2 \\
\hline Reduced activity & 17 & 9.4 \\
\hline Drug shortage & 30 & 16.7 \\
\hline Drug incompliance & 19 & 10.6 \\
\hline \multicolumn{3}{|l|}{ Contacting your physician } \\
\hline Less than usual & 80 & 44.4 \\
\hline As usual & 44 & 24.4 \\
\hline More than usual & 5 & 2.8 \\
\hline I did not & 51 & 28.3 \\
\hline \multicolumn{3}{|l|}{ Means of communication } \\
\hline Phone call & 29 & 16.1 \\
\hline Clinic & 50 & 27.8 \\
\hline Internet & 63 & 35 \\
\hline Send someone & 2 & 1.1 \\
\hline I did not & 36 & 20 \\
\hline \multicolumn{3}{|l|}{ Cause of communication } \\
\hline Disease activity or pain & 44 & 24.4 \\
\hline COVID-19 symptoms & 5 & 2.8 \\
\hline Drug shortage & 24 & 13.3 \\
\hline Medication advice & 51 & 28.3 \\
\hline Ask about COVID-19 & 6 & 3.3 \\
\hline I did not & 50 & 27.8 \\
\hline \multicolumn{3}{|l|}{${ }^{a}$ beeling after communication } \\
\hline Feel more anxiety and fear & 14 & 7.8 \\
\hline Feel the same & 75 & 41.7 \\
\hline $\mathrm{a}$ b Feel less anxiety and fear & 37 & 20.6 \\
\hline a did not & 54 & 30 \\
\hline Fear of drugs (no/yes) & $108 / 72$ & $60 / 40$ \\
\hline a b Stopped drugs (no/yes) & $148 / 32$ & $82.2 / 17.8$ \\
\hline a'Drug shortage (no/yes) & $57 / 123$ & $31.7 / 68.3$ \\
\hline Reduce doses (no/yes) & $122 / 58$ & $67.8 / 32.2$ \\
\hline Physician advice if you have COVID (no/yes) & $152 / 28$ & $84.4 / 15.6$ \\
\hline
\end{tabular}

${ }^{a}$ Significant between RA, SLE, others

${ }^{\mathrm{b}}$ Significant between RA, SLE. COVID-19; Coronavirus disease 2019 
Table 5 Relation between different parameters and patients' mental health ( $n=180)$

\begin{tabular}{|c|c|c|c|c|}
\hline Parameters & BSRS-5 < $6(n=26)$, no $(\%)$ & BSRS-5 $\geq 6(n=154)$, no $(\%)$ & ${ }^{a}$ Test & $p$ value \\
\hline Age (years) $\leq$ median $(34)(n=97)$ & $12(12.4 \%)$ & $85(87.6 \%)$ & 0.732 & 0.392 \\
\hline Female sex $(n=141)$ & $18(12.8 \%)$ & $123(87.2 \%)$ & 1.484 & 0.223 \\
\hline Non-smoker $(n=150)$ & $22(14.7 \%)$ & $128(85.3 \%)$ & Fisher & 0.850 \\
\hline \multicolumn{5}{|l|}{ COVID-19 information source } \\
\hline TV/radio $(n=39)$ & $6(15.4 \%)$ & $33(84.6 \%)$ & \multirow[t]{5}{*}{5.927} & \multirow[t]{5}{*}{0.205} \\
\hline Internet $(n=42)$ & $9(21.4 \%)$ & $33(78.6 \%)$ & & \\
\hline Social media $(n=88)$ & $10(11.4 \%)$ & $78(88.6 \%)$ & & \\
\hline My physician $(n=9)$ & $0.0(00 \%)$ & $9(100 \%)$ & & \\
\hline Friends and family $(n=2)$ & $1(50 \%)$ & $1(50 \%)$ & & \\
\hline \multicolumn{5}{|l|}{ Rheumatological disease } \\
\hline $\mathrm{RA}(n=72)$ & $16(22.2 \%)$ & $56(77.8 \%)$ & \multirow[t]{3}{*}{5.887} & \multirow[t]{3}{*}{0.053} \\
\hline $\operatorname{SLE}(n=67)$ & $6(9 \%)$ & $61(91 \%)$ & & \\
\hline Others $(n=41)$ & $4(9.8 \%)$ & $37(90.2 \%)$ & & \\
\hline No lung affection $(n=141)$ & $20(14.2 \%)$ & $121(85.8 \%)$ & 0.036 & 0.850 \\
\hline \multicolumn{5}{|c|}{ Means of protection: Staying at home } \\
\hline No $(n=18)$ & $5(27.8 \%)$ & $13(72.2 \%)$ & \multirow[t]{3}{*}{2.895} & \multirow[t]{3}{*}{0.235} \\
\hline Yes $(n=98)$ & $13(13.3 \%)$ & 85 (86.7\%) & & \\
\hline Maybe $(n=64)$ & $8(18.5 \%)$ & $56(87.5 \%)$ & & \\
\hline \multicolumn{5}{|l|}{ Wearing masks } \\
\hline No $(n=52)$ & $6(11.5 \%)$ & $46(88.5 \%)$ & \multirow[t]{3}{*}{0.710} & \multirow[t]{3}{*}{0.701} \\
\hline Yes $(n=57)$ & $8(14 \%)$ & $49(86 \%)$ & & \\
\hline Maybe $(n=71)$ & $12(16.9 \%)$ & $59(83.1 \%)$ & & \\
\hline \multicolumn{5}{|l|}{ Wearing gloves } \\
\hline No $(n=79)$ & $14(17.7 \%)$ & $65(82.3 \%)$ & \multirow[t]{3}{*}{1.250} & \multirow[t]{3}{*}{0.535} \\
\hline Yes $(n=36)$ & $4(11.1 \%)$ & $32(88.9 \%)$ & & \\
\hline May be $(n=65)$ & $8(12.3 \%)$ & $57(87.7 \%)$ & & \\
\hline \multicolumn{5}{|l|}{ Washing hands } \\
\hline No $(n=3)$ & $0.0(00 \%)$ & $3(100 \%)$ & \multirow[t]{3}{*}{1.394} & \multirow[t]{3}{*}{0.498} \\
\hline Yes $(n=129)$ & $17(13.2 \%)$ & $112(86.8 \%)$ & & \\
\hline Maybe $(n=48)$ & $9(18.8 \%)$ & 39 (81.2\%) & & \\
\hline \multicolumn{5}{|l|}{ Healthy diet and exercises help } \\
\hline No $(n=21)$ & $2(9.5 \%)$ & $19(90.5 \%)$ & \multirow[t]{3}{*}{3.301} & \multirow[t]{3}{*}{0.192} \\
\hline Yes $(n=95)$ & $18(18.9 \%)$ & 77 (81.1\%) & & \\
\hline Maybe $(n=64)$ & $6(9.4 \%)$ & $58(90.6 \%)$ & & \\
\hline \multicolumn{5}{|l|}{ Going outdoors } \\
\hline Never $(n=48)$ & $8(16.7 \%)$ & $40(83.3 \%)$ & \multirow[t]{3}{*}{1.249} & \multirow[t]{3}{*}{0.535} \\
\hline Only for necessities ( $n=113$ ) & $14(12.4 \%)$ & 99 (87.6\%) & & \\
\hline Go out freely $(n=19)$ & $4(21.1 \%)$ & $15(78.9 \%)$ & & \\
\hline Wearing mask outdoors & & & & \\
\hline Never $(n=66)$ & $11(16.7 \%)$ & $55(83.3 \%)$ & 3.013 & 0.556 \\
\hline Few times $(n=28)$ & $5(17.9 \%)$ & $23(82.1 \%)$ & & \\
\hline Most of the time $(n=31)$ & $2(6.5 \%)$ & $29(93.5 \%)$ & & \\
\hline Always ( $n=22$ ) & $2(9.1 \%)$ & $20(90.9 \%)$ & & \\
\hline I do not go out $(n=33)$ & $6(18.2 \%)$ & $27(81.8 \%)$ & & \\
\hline
\end{tabular}


Table 5 Relation between different parameters and patients' mental health $(n=180)$ (Continued)

\begin{tabular}{|c|c|c|c|c|}
\hline Parameters & BSRS-5 < $6(n=26)$, no $(\%)$ & BSRS-5 $\geq 6(n=154)$, no $(\%)$ & ${ }^{a}$ Test & $p$ value \\
\hline \multicolumn{5}{|l|}{ Wearing loves outdoors } \\
\hline Never $(n=90)$ & $16(17.8 \%)$ & $74(82.2 \%)$ & 3.474 & 0.482 \\
\hline Few times $(n=21)$ & $2(9.5 \%)$ & 19 (90.5\%) & & \\
\hline Most of the time $(n=20)$ & $1(5 \%)$ & 19 (95\%) & & \\
\hline Always $(n=14)$ & $1(7.1 \%)$ & $13(92.9 \%)$ & & \\
\hline I do not go out $(n=35)$ & $6(17.1 \%)$ & 29 (82.9\%) & & \\
\hline \multicolumn{5}{|l|}{ Staying in my room } \\
\hline Move freely at home $(n=133)$ & $23(17.3 \%)$ & $110(82.7 \%)$ & 4.597 & 0.204 \\
\hline A bit of time $(n=19)$ & $0.0(00 \%)$ & 19 (100\%) & & \\
\hline Most of time $(n=26)$ & $3(11.5 \%)$ & $23(88.5 \%)$ & & \\
\hline All of time $(n=2)$ & $0.0(00 \%)$ & $2(100 \%)$ & & \\
\hline \multicolumn{5}{|l|}{ Frequency washing hands } \\
\hline Less than 5 times $(n=31)$ & $3(9.7 \%)$ & $28(90.3 \%)$ & 0.689 & 0.709 \\
\hline 5 to 10 times $(n=97)$ & $15(15.5 \%)$ & $82(84.5 \%)$ & & \\
\hline More than 10 times $(n=52)$ & 8 (15.4\%) & 44 (84.6\%) & & \\
\hline
\end{tabular}

COVID-19 coronavirus disease 2019, BSRS-5 5-item Brief Symptom Rating Scale

${ }^{a}$ Chi square test $\left(x^{2}\right)$

${ }^{\mathrm{b}}$ Cell of significance

Also, comparing between patients with RA and SLE only, a statistically significant difference $[p \leq 0.05]$ was found among them as regards some parameters. RA patients had higher mean age, lower mean BSRS-5 total score, and a higher percentage of the male sex, "yes healthy diet and exercises help," feel "not at all/quite a bit inferiority," feel "maybe/not at risk," feel less anxiety and fear after communication, and stopped drugs more often.

\section{Discussion}

COVID-19 pandemic has affected people's lives in many different aspects. The fact that neither vaccine nor confirmed effective treatment has been developed has made the Coronavirus a huge stressor and a fearful threat to many individuals [23]. With COVID-19, the social isolation, quarantine, and loneliness in addition to fear of infection or death were the main factors impacting not only those infected with COVID but also the general population. The resulting fears, although considered a usual response to this major threatening event, when chronic and profound becomes harmful and may lead to psychiatric illness [24, 25].

Health professionals, including doctors and nurses, being the most at risk, suffered impaired mental health $[26,27]$. Special population groups who are considered more vulnerable to infection- old age, immunecompromised, and patients with chronic illnesses-frequently complained of considerable psychological problems [7]. The "double crush" of having a chronic illness and receiving immunosuppressive drugs makes patients with rheumatic diseases among these groups more vulnerable to infection and thus impaired mental health.

Most patients were non-smokers and the majority had no previous lung affection. However, smokers and those with lung affection did not seem to have a different attitude or behavior regarding being at a greater risk, most probably due to the fact that most patients with chronic diseases had comparable degrees of anxiety. When comparing the attitude and behavior towards COVID among the studied groups, no statistically significant difference was found between cases and control group regarding their attitude towards staying at home, the value of a healthy diet and exercises and washing hands; while there was a statistically significant difference between both groups regarding going outdoors, also, wearing masks and gloves outdoors, where patients seemed to be more selfprotecting. Self-isolation in their room most of the time was mostly practiced by patients more than controls.

Previous studies have shown that mental health disorders can last longer than the epidemic itself $[8,9]$. Individuals with chronic illnesses are even more exposed to psychological impairment [16, 17]. Mental health comparison among the studied groups using the BSRS showed that there was a statistically significant difference between both groups regarding "feeling angry/irritated," "feeling inferior to others," insomnia, and regarding the BSRS total score. Out of all patients, $85.6 \%$ were defined as a psychiatric case. Their belief of "being at risk" was associated with a higher degree of impaired mental health [BSRS-5 $\geq 6$ ]. These distress reactions [feeling angry, irritable, and disturbed sleep] being more 
Table 6 Relation between parameters and patients mental health degree $(n=180)$

\begin{tabular}{|c|c|c|c|c|}
\hline Parameters & BSRS-5 < $6(n=26)$, no (\%) & BSRS-5 $\geq 6(n=154)$, no $(\%)$ & ${ }^{a}$ Test & $p$ value \\
\hline \multicolumn{5}{|l|}{ Your fear about } \\
\hline Infection $(n=26)$ & $3(11.5 \%)$ & $23(88.5 \%)$ & 8.320 & 0.139 \\
\hline Family $(n=92)$ & $16(17.4 \%)$ & $76(82.6 \%)$ & & \\
\hline Quarantine $(n=6)$ & $0.0(00 \%)$ & $6(100 \%)$ & & \\
\hline Financial problems $(n=14)$ & $1(7.1 \%)$ & $13(92.9 \%)$ & & \\
\hline Medications $(n=11)$ & $4(36.4 \%)$ & $7(63.6 \%)$ & & \\
\hline Mixed fears $(n=31)$ & $2(6.5 \%)$ & 29 (93.5\%) & & \\
\hline \multicolumn{5}{|l|}{ Are you at risk } \\
\hline No $(n=23)$ & $6(26.1 \%)$ & $17(73.9 \%)$ & 7.036 & $0.030^{*}$ \\
\hline Yes $(n=97)$ & b8 (8.2\%) & $89(91.8 \%)$ & & \\
\hline Maybe $(n=60)$ & $12(20 \%)$ & $48(80 \%)$ & & \\
\hline \multicolumn{5}{|l|}{ Current disease activity } \\
\hline Decreased $(n=6)$ & $1(16.7 \%)$ & $5(83.3 \%)$ & 1.211 & 0.546 \\
\hline Did not change $(n=131)$ & $21(16 \%)$ & $110(84 \%)$ & & \\
\hline Increased $(n=43)$ & $4(9.3 \%)$ & 39 (90.7\%) & & \\
\hline \multicolumn{5}{|l|}{ Activity may increase due to } \\
\hline Fear $(n=92)$ & $9(9.8 \%)$ & $83(90.2 \%)$ & 5.096 & 0.278 \\
\hline Postponed follow-up visits ( $n=22$ ) & $4(18.2 \%)$ & $18(81.8 \%)$ & & \\
\hline Reduced activity $(n=17)$ & $5(29.4 \%)$ & $12(70.6 \%)$ & & \\
\hline Drug shortage $(n=30)$ & $5(16.7 \%)$ & $25(83.3 \%)$ & & \\
\hline Drug incompliance $(n=19)$ & $3(15.8 \%)$ & $16(84.2 \%)$ & & \\
\hline \multicolumn{5}{|l|}{ Contact physician } \\
\hline Less than usual $(n=80)$ & $13(16.2 \%)$ & $67(83.8 \%)$ & 1.428 & 0.699 \\
\hline As usual $(n=44)$ & 7 (15.9\%) & $37(84.1 \%)$ & & \\
\hline More than usual $(n=5)$ & $0.0(00 \%)$ & $5(100 \%)$ & & \\
\hline I did not $(n=51)$ & $6(11.8 \%)$ & $45(88.2 \%)$ & & \\
\hline \multicolumn{5}{|l|}{ Means of communication } \\
\hline Phone call $(n=29)$ & $4(13.8 \%)$ & $25(86.2 \%)$ & 3.631 & 0.458 \\
\hline Clinic $(n=50)$ & $6(12 \%)$ & $44(88 \%)$ & & \\
\hline Internet $(n=63)$ & $13(20.6 \%)$ & $50(79.4 \%)$ & & \\
\hline Send someone $(n=2)$ & $0.0(00 \%)$ & $2(100 \%)$ & & \\
\hline I did not $(n=36)$ & $3(8.3 \%)$ & $33(91.7 \%)$ & & \\
\hline \multicolumn{5}{|l|}{ Cause of communication } \\
\hline Disease activity or pain $(n=44)$ & $4(9.1 \%)$ & 40 (90.9\%) & 5.575 & 0.350 \\
\hline COVID symptoms $(n=5)$ & $0.0(00 \%)$ & $5(100 \%)$ & & \\
\hline Drug shortage $(n=24)$ & $5(20.8 \%)$ & 19 (79.2\%) & & \\
\hline Medication advice $(n=51)$ & $11(21.6 \%)$ & $40(78.4 \%)$ & & \\
\hline Ask about COVID $(n=6)$ & $1(16.7 \%)$ & $5(83.3 \%)$ & & \\
\hline I did not $(n=50)$ & $5(10 \%)$ & 45 (90\%) & & \\
\hline \multicolumn{5}{|l|}{ Feeling after communication } \\
\hline Feel more anxiety and fear $(n=14)$ & $1(7.1 \%)$ & $13(92.9 \%)$ & 3.985 & 0.263 \\
\hline Feel the same $(n=75)$ & $9(12 \%)$ & $66(88 \%)$ & & \\
\hline Feel less anxiety and fear $(n=37)$ & $9(24.3 \%)$ & $28(75.7 \%)$ & & \\
\hline I did not $(n=54)$ & $7(13 \%)$ & 47 (87\%) & & \\
\hline
\end{tabular}


Table 6 Relation between parameters and patients mental health degree $(n=180)$ (Continued)

\begin{tabular}{|c|c|c|c|c|}
\hline Parameters & BSRS-5 < $6(n=26)$, no (\%) & BSRS-5 $\geq 6(n=154)$, no (\%) & ${ }^{a}$ Test & $p$ value \\
\hline Fear of drugs $(n=108)$ & $20(18.5 \%)$ & $88(81.5 \%)$ & 3.626 & 0.057 \\
\hline Stopped drugs $(n=148)$ & $24(16.2 \%)$ & $124(83.8 \%)$ & Fisher & 0.146 \\
\hline Experienced drug shortage $(n=123)$ & $17(13.8 \%)$ & $106(86.2 \%)$ & 0.122 & 0.727 \\
\hline Reduced doses of drugs $(n=122)$ & $18(14.8 \%)$ & $104(85.2 \%)$ & 0.029 & 0.864 \\
\hline
\end{tabular}

COVID-19 coronavirus disease 2019, BSRS-5 5-item Brief Symptom Rating Scale

${ }^{\mathrm{a}} \mathrm{Chi}$ square test $\left(x^{2}\right)$

${ }^{\mathrm{b}}$ Cell of significance

* $P$ value $<0.05$ is significant

prominent in cases denote fear and panic. About 20\% were "extremely" angry and agitated. Moreover, cases were feeling more inferior, which is most probably related to their chronic illness. Ironically, no significant difference was found regarding anxiety and depression between cases and controls denoting that the general population during the pandemic felt similar degrees of anxiety and depression. Fear of infection or death raises anxiety levels in healthy individuals as well, quarantine and social isolation increased depression in both groups similarly. Both patients and healthy feared mostly losing their families and beloved ones.

Regarding the attitude and behavior of the cases towards medications; the highest proportion of them answered that they have no fear of drugs, they did not reduce doses, nor did they stop any of their drugs. Those who stopped drugs may have had financial issues or difficulty finding medications. This is a good positive attitude consistent with the EULAR guidelines regarding medications, representing their awareness of the necessity of continuing their medications to avoid flares [28]. Moreover, many of them did not experience any increase in their disease activity, although many believed that fear itself may cause a flare. The most frequent cause to contact their physician was medication advice rather than consultation due to pain and disease activity. The increased usage of antimalarial drugs to treat COVID-19 patients caused a decline in their availability in pharmacies, highlighting the harm to patients caused by medications being purchased in Egypt without medical prescription [29]. For some patients, this might be an essential treatment especially for those with SLE, and many had to communicate with their physicians for substitutes. During the pandemic, most of the patients visited their physicians less than usual to minimize going outdoors to healthcare facilities; they mostly contacted them using telehealth.

Regarding the comparison between patients with RA, SLE, and other rheumatic diseases, RA patients had higher mean age and a higher percent of the male sex, while SLE patients were mostly younger females. Most RA patients believe that a healthy diet and exercise can help combat infection while SLE patients are less confident about that. RA patients only go out for necessities while most SLE patients never go outdoors as they felt that they are at risk more than other patients. They even feel more/the-same anxiety and fear after communicating with their physicians. Most of SLE patients are adherent to their drugs more than other patients. We conclude that although rheumatologists succeeded to convince their patients to stick to their medications, they failed to relieve their anxiety and fear which may be augmented by media, following numbers of those COVIDinfected and dead. About 48\% depended on social media as a source of information about COVID-19. Both RA patients and SLE patients experienced drug shortages. Most patients with other rheumatic diseases go outdoors more freely, they do not feel great that they are at a higher risk like SLE patients nor do they significantly complain of drug shortages as they are less dependent on anti-malarials unlike SLE patients.

Regarding their mental status RA patients had significantly lower mean BSRS-5 total score and felt less inferiority than SLE patients. No significant difference was found in patients with different diseases regarding anxiety and depression. Although cases were significantly different regarding anger and insomnia compared to controls, there were no differences comparing patients with different disease categories.

The limitations of our study were the inability to assess the same patients' psychological status just before the hit of coronavirus, an unexpected event, which would have been more informative about the direct impact of COVID-19 pandemic isolated from the effect of their chronic illnesses which had been also accused to affect mental health [30, 31]. The finding that mental health status was more affected by their belief of "being at risk" of infection was a clear indicator of the impact of COVID-19 pandemic. The presented data are preliminary data that could be obtained during the quarantine period and we are planning for future studies to test behavioral changes on rheumatic patients that had suffered from COVID19 infection. Another limitation is recruiting patients who use social media with no inclusion of patients not using it as illiterate patients and patients from 
low socioeconomic that are not familiar with social media.

\section{Conclusion}

Patients with rheumatic diseases show comparable degrees of anxiety and depression to healthy individuals, but higher distress symptoms and panic in the form of anger, irritability, and insomnia. They have a significantly higher sense of inferiority and a higher total BSRS compared to controls. SLE patients show more adherence to their medications and stay mostly at home as a reflection of feeling more vulnerable. Moreover, they have higher degrees of psychological affection in the form of higher BSRS scores. Abandoning drug purchasing without medical prescription is necessary in Egypt to protect our patients from unnecessary drug shortages adding to their fear and anxiety. Mental health should be addressed in the same manner we deal with the infectious disease itself, being of no less importance. Mental health professionals, social workers, and support groups need to provide psychological support to vulnerable populations, including patients with rheumatic diseases. Rheumatologists should be aware of the need for psychiatric consultation for their patients whenever necessary.

\section{Supplementary Information}

The online version contains supplementary material available at https://doi. org/10.1186/s43166-020-00045-y.

\section{Additional file 1.}

\section{Abbreviations \\ COVID-19: Coronavirus disease 2019; BSRS-5: Brief Symptom Rating Scale; SARS-CoV-2: Severe acute respiratory syndrome coronavirus 2; WHO: World Health Organization; ACR: American College of Rheumatology; IRB: Institutional review board; RA: Rheumatoid arthritis; SLE: Systemic lupus erythematosus}

\section{Acknowledgements}

Not applicable.

\begin{abstract}
Authors' contributions
MAHH: substantial contributions to the conception, design of the work; the acquisition, analysis, and interpretation of data for the work. Drafting the work and revising it critically for important intellectual content. Final approval of the version to be published. Agreement to all aspects of the work. ME: substantial contributions to the acquisition, analysis, and interpretation of data for the work. Drafting the work and revising it critically for important intellectual content. Final approval of the version to be published. Agreement to all aspects of the work. GAD: Substantial contributions interpretation of data for the work, revision of the work critically for important intellectual content, final approval of the version to be published, agreement to all aspects of the work. All authors read and approved the final manuscript.
\end{abstract}

\section{Funding}

No specific funding was received from anybody in the public, commercial or non-profit organizations.

Availability of data and materials

Available upon request.
Ethics approval and consent to participate

Ethical approval was taken from IRB of Zagazig University under the number of ZUIRB\#6096. Online consent was taken from all participants.

\section{Consent for publication}

Not applicable.

\section{Competing interests}

The authors declare no conflicts of interest.

\section{Author details}

${ }^{1}$ Rheumatology and Rehabilitation Department, Faculty of Medicine, Zagazig University, Zagazig, Egypt. ${ }^{2}$ Rheumatology Department, Faculty of Medicine, Cairo University, Cairo, Egypt.

Received: 7 August 2020 Accepted: 29 October 2020

Published online: 12 November 2020

\section{References}

1. Lu H, Stratton CW, Tang Y-W (2020) Outbreak of pneumonia of unknown etiology in Wuhan, China: the mystery and the miracle. J Med Virol 92(4): 401-402 Available from: https://pubmed.ncbi.nlm.nih.gov/31950516

2. Who.com. World Health Organization. Available from: https://time.com/5 791661/who-coronavirus-pandemic-declaration. cited May, 242020

3. Wu KK, Chan SK, Ma TM (2005) Posttraumatic stress, anxiety, and depression in survivors of severe acute respiratory syndrome [SARS]. J Trauma Stress 18(1):39-42 Available from: https://pubmed.ncbi.nlm.nih.gov/16281194

4. Chong M-Y, Wang W-C, Hsieh W-C, Lee C-Y, Chiu N-M, Yeh W-C et al (2004) Psychological impact of severe acute respiratory syndrome on health workers in a tertiary hospital. Br J Psychiatry 185(2):127-133. Available from. https://doi.org/10.1192/bjp.185.2.127

5. Goulia P, Mantas C, Dimitroula D, Mantis D, Hyphantis T (2010) General hospital staff worries, perceived sufficiency of information and associated psychological distress during the a/H1N1 influenza pandemic. BMC Infect Dis 10:322 Available from: https://pubmed.ncbi.n/m.nih.gov/21062471

6. Docea AO, Tsatsakis A, Albulescu D, Cristea O, Zlatian O, Vinceti M et al (2020) A new threat from an old enemy: re-emergence of coronavirus [review]. Int J Mol Med 45(6):1631-1643 Available from: https://pubmed. ncbi.nlm.nih.gov/32236624

7. Xiang Y-T, Yang Y, Li W, Zhang L, Zhang Q, Cheung T et al (2020) Timely mental health care for the 2019 novel coronavirus outbreak is urgently needed. Lancet Psychiatry 7(3):228-229 Available from: https://pubmed.ncbi. nlm.nih.gov/32032543

8. Reardon S (2015) Ebola's mental-health wounds linger in Africa. Nature 519(7541):13-14. Available from. https://doi.org/10.1038/519013a

9. Shigemura J, Ursano RJ, Morganstein JC, Kurosawa M, Benedek DM (2020) Public responses to the novel 2019 coronavirus [2019-nCoV] in Japan: mental health consequences and target populations. Psychiatry Clin Neurosci 74(4):281-282 Available from: https://pubmed.ncbi.nlm.nih.gov/32 034840

10. Malta M, Rimoin AW, Strathdee SA (2020) The coronavirus 2019-nCoV epidemic: is hindsight 20/20? E Clinical Medicine 3(20):100289 Available from: https://pubmed.ncbi.nlm.nih.gov/32154505

11. Cascella M, Rajnik M, Cuomo A, Dulebohn SC, Di Napoli R (2020) Features, evaluation and treatment coronavirus [COVID-19]. StatPearls Publishing Stat Pearls Publishing LLC, Treasure Island

12. Peeri NC, Shrestha N, Rahman MS. et al. (2020) The SARS, MERS and novel coronavirus (COVID-19) epidemics, the newest and biggest global health threats: what lessons have we learned? Int J Epidemiol 1;49(3):717-726. https://doi.org/10.1093/ije/dyaa033.

13. Ornell F, Schuch JB, Sordi AO, Kessler FHP (2020) "pandemic fear" and COVID-19: mental health burden and strategies. Rev Bras Psiquiatr 42(3): 232-235 Available from: https://pubmed.ncbi.nlm.nih.gov/3226734314

14. Buheji M, Buheji A (2020) Planning competency in the new Normalemployability competency in post- COVID-19 pandemic. Int J Hum Resour Stud 10(2):237. Available from:. https://doi.org/10.5296/ijhrs.v10i2.17085

15. Li W, Yang Y, Liu Z-H, Zhao Y-J, Zhang Q, Zhang L, Cheung T, Xiang Y-T (2020) Progression of mental health services during the COVID-19 outbreak in China. Int J Biol 16(10):1732-1738 
16. Applegate WB, Ouslander JG (2020) COVID-19 presents high risk to older persons. J Am Geriatr Soc 68(4):681 Available from: https://pubmed.ncbi. nlm.nih.gov/32154911

17. Dong XC, Li JM, Bai JY, Liu ZQ, Zhou PH, Gao L et al (2020) Epidemiological characteristics of confirmed COVID-19 cases in Tianjin. Zhonghua Liu Xing Bing Xue Za Zhi 41:638-642

18. Mikuls TR, Johnson SR, Fraenkel L, Arasaratnam RJ, Baden LR, Bermas BL, Saag KG (2020) American College of Rheumatology Guidance for the Management of Adult Patients with rheumatic disease during the COVID-19 pandemic. Arthritis Rheumatol. Available from. https://doi.org/10.1002/art. 41301

19. Wang C, Pan R, Wan X, Tan Y, Xu L, Ho CS et al (2020) Immediate psychological responses and associated factors during the initial stage of the 2019 coronavirus disease [COVID-19] epidemic among the general population in China. Int J Environ Res Public Health 17(5):1729 Available from: https://pubmed.ncbi.nlm.nih.gov/32155789

20. Lee H, Han J, Kang J (2020. Available from:) Validity and reliability of the Korean anesthesia surrendering instrument. Res Square. https://doi.org/10. 21203/rs.2.22075/v2

21. Chen HC, Wu CH, Lee YJ et al (2005) Validity of the five-item brief symptom rating scale among subjects admitted for general health screening. J Formos Med Assoc 104:824-829

22. Lung FW, Lee MB (2008) The five-item brief-symptom rating scale as a suicide ideation screening instrument for psychiatric inpatients and community residents. BMC Psychiatry 8:53-60

23. Hawryluck L, Gold WL, Robinson S, Pogorski S, Galea S, Styra R (2004) SARS control and psychological effects of quarantine, Toronto, Canada. Emerg Infect Dis 10(7):1206-1212 Available from: https://pubmed.ncbi.nlm.nih. gov/15324539

24. Garcia R (2017) Neurobiology of fear and specific phobias. Learn Mem 24(9): 462-471 Available from: https://pubmed.ncbi.nlm.nih.gov/2881447226

25. Shin LM, Liberzon I (2010) The neurocircuitry of fear, stress, and anxiety disorders. Neuropsychopharmacology 35(1):169-191 Available from: https:// pubmed.ncbi.nlm.nih.gov/19625997

26. Kang L, Li Y, Hu S, Chen M, Yang C, Yang BX et al (2020) The mental health of medical workers in Wuhan, China dealing with the 2019 novel coronavirus. Lancet Psychiatry 7:e14

27. Huang JZ, Han MF, Luo TD, Ren AK, Zhou XP. (2020) [Mental health survey of medical staff in a tertiary infectious disease hospital for COVID-19]. Zhonghua Lao Dong Wei Sheng Zhi Ye Bing Za Zhi. 20;38(3):192-195. Chinese. https://doi.org/10.3760/cma.j.cn121094-20200219-00063.

28. EULAR | EULAR Guidance for patients COVID-19 outbreak. Available from: https://www.eular.org/eular_guidance_for_patients_covid19_outbreak.cfm. Cited May 24, 2020

29. Spinelli FR, Ceccarelli F, Di Franco M, Conti F (2020) Response to 'To consider or not antimalarials as a prophylactic intervention in the SARSCov-2 [Covid-19] pandemic' by Parperis. Ann Rheum Dis:2020-217634. Available from:. https://doi.org/10.1136/annrheumdis-2020-2176

30. Lwin MN, Serhal L, Holroyd C, Edwards CJ (2020) Rheumatoid arthritis: the impact of mental health on disease: a narrative review. Rheumatol Ther 7 : 457-471. Available from. https://doi.org/10.1007/s40744-020-00217-4

31. Dregan A, Matcham F, Harber-Aschan L, Rayner L, Brailean A, Davis K, Hatch S, Pariante C, Armstrong D, Stewart R, Hotopf M (2019) Epidemiology (common mental disorders within chronic inflammatory disorders: a primary care database prospective investigation). Ann Rheum Dis 78(5):688695 Available from https:/onlinelibrary.wiley.com/doi/full/10.1002/acr.23539

\section{Publisher's Note}

Springer Nature remains neutral with regard to jurisdictional claims in published maps and institutional affiliations.

\section{Submit your manuscript to a SpringerOpen ${ }^{\circ}$ journal and benefit from:}

- Convenient online submission

- Rigorous peer review

- Open access: articles freely available online

High visibility within the field

- Retaining the copyright to your article

Submit your next manuscript at $\boldsymbol{\nabla}$ springeropen.com 